fledged science. At the beginning of this period, astrophysics was almost non-existent. Thus the first lecture printed here is Airy's discourse on the fortheoming solar eclipse of 1851. This was the first to bo observed in detail by astronomers, and it led to the long sequence of nineteonth century eclipse expeditions, which raised so many questions concerring the nature of the solar atmosphere -and answered a few of them. At the other end of the time sequence, the last discourse is by another Astronomer Royal, Spencer Jones, on the projected 200-inch telescope: the instrument which better than any other symbolizes modern astrophysics.

These volumes allow us to follow the often complicated development of astrophysical ideas during the past century. Many of the conceptual changes have been remarkable: none more so than in the timescale allotted to the universe. In 1887, Sir William Thomson explained to his audience: "Five or ten million years ago he (the Sun) may have been about double his present diameter and an eighth of his present mean density ... but we cannot, with any probability of argument or speculation, go on continuously much beyond that". Just about fifty years later Sir James Jeans told his audience: "... we find that 5 million million years ago, the Sun was probably many times as massive as now and many times as bright". The current value for the age of the Sun is more or less the geometric mean of these two estimates.

The timescale has been a central question in astronomy throughout the nineteenth and twentieth centuries; other areas, less subject to intense scrutiny, have experienced fluctuations in fashion. Dewar's experiments on the gaseous constituents of meteorites-reported by him to the Royal Institution in 1886 -were for many years the best of their kind: it is only in the past two decades that there has been a renewed interest in this type of investigation. Similarly, there have been varying fashions in ideas: Balfour Stewart's discourse (1867) on the Sun as a variable star refers to the part played by the planets in influencing the behaviour of sunspots. This suggestion was revived, albeit in modified form, just a century later, in the 1960s. In view of the current British plans for the development of infrared astronomy, Piazzi Smyth's preliminary site-testing in Teneriffe (reported to the Royal Institution in 1858) may also come in useful after a lapse of a century. Smyth, happily, was enthusiastic about the site. "Then how truly serene and quiet, and transparent, too, was the air above our 8,900 foot elevation; for, on erecting our telescopes, not only was each star, high or low, seen with an exquisite little disc and ncarly perfect rings, but the space-penetrating power was extended with the same instrument and same eye from the 10th magnitude at the sea level, to the 14th."

These two volumes are fascinating, if rather expensive, and can be recommended both for serious and for bedtime reading. Misprints in the body of the text cannot, of course, be blamed on the publishers, because these are photographic reprints. But the blurb describes the editor, Sir Bernard Lovell, as having been president of the Royal Astronomical Society in 1964; he is in fact the current president.

A. J. Meadows

\section{RADIO ASTROPHYSICS}

\section{Radio Astrophysies}

Nonthermal Processes in Galactic and Extragalactic Sources. By A. G. Pacholezyk. (Series of Books in Astronomy and Astrophysics.) Pp. xxi +269 . (Freoman: San Franciseo and Folkestone, September 1970.) 122s.

A LARGE body of observational data is available for the 10,000 or so radio sources now known. Theso include, among other parameters, measures of source spectra, energy flux, sizo and polarization. Before this material can be interpreted in terms of the physical conditions within the sources, it is necessary to have a well understood theory of the emission mechanism. Indeed, the synchrotron mechanism in which radiation is generated by relativistic electrons gyrating in a magnetic field is believed to be an adequate theory for the majority, if not all, of the galactic and extragalactic continuum radio sources.

Dr Pacholczyk has written a usoful text on the emission processes in radio sources. He has concentrated on the synchrotron process for which he has provided a thorough introduction. The effects on the emission by the surrounding plasma are included as are the effects of electron scattering on the emitted synchrotron radiation. A well chosen bibliography is included at the end of each chapter.

A new range of non-thermal processes have recently been recognized in spectral line sources. The most studied of those are the regions of anomalous emission from $\mathrm{OH}$ and $\mathrm{H}_{2} \mathrm{O}$ molecules in interstellar space. Brightness temperatures of up to $10^{13} \mathrm{~K}$ have been measured which are enormously in excess of the kinetic temperatures which exist in these regions and are interpreted as originating in maser action where a weak background signal is amplified in the cloud of interstellar molecules to these high intensities. Line-narrowing, temporal variability and a high degree of circular polarization are trade marks of this type of emission.

It is probably a sign of the rate of advance technically and the associated rate of new discoveries in radioastronomy that this subject is scarcely touched on in Pacholczyk's treatment. A major branch of non-thermal radio emission mechanisms has been deliberately excluded from this work, namely, the various radio emission mechanisms known to operate in the atmosphere of the Sun. The reason for this latter omission in not so clear, because these mechanisms provide excellent examples of emission mechanisms other than the synchrotron process.

In all, I find this a welcome book bridging the gap between the extensive theoretical treatments and the wide range of observational data. As an aside, it is interesting to find Pacholczyk, a theoretical astrophysicist, falling prey to the observational astronomers' and spacescientists' nightmare-confusing the senses of polarization which an antenna receives and transmits. An antenna receives and transmits the same sense of circular polarization as is adequately demonstrated in Kraus's Radioastronomy.

R. D. Davtes

\section{THE FACE OF THE MOON}

\section{Geology of the Moon}

A Stratigraphic View. By Thomas A. Mutch. Pp. 324. (Princeton University: Princeton, New Jersey; Oxford University: London, August 1970.) 165s.

Professor Mutch, a stratigrapher who has worked at the US Geological Survey's Center of Astrogeology at Flagstaff, is well qualified to discuss lunar stratigraphy. His book is not, however, as specialized as the subtitle might indicate. The author appeals to all the important aspects of lunar geology, albeit on a superficial level. The text is relatively free from prejudice and, together with the general lack of great depth of treatment, combines with the well-chosen illustrations to provide an excellent students' introduction to the geology of the Moon.

Two introductory chapters on aspects of history of the subject and the Moon's orbit and figure are brief and more adequate treatments will be found elsewhere. The third introductory chapter deals, again briefly, with photographic documentation of the Moon and the results drawn from some of the physical methods (such as optical and thermal) used to examine the lunar surface. Key charts identify the locations of the Lunar Orbiters' photographic coverage (but neither cardinal points nor coordinates are 
given on the full Moon charts). It is useful to be reminded how the Orbiter photographic strips were assembled from scans of the $70 \mathrm{~mm}$ film carried aboard the spacecraft.

Lunar photographs are used liberally-and are among the best ever taken. They are pleasingly reproduced, with a matt finish; but one could wish for better because many of them tend to be rather "muddy".

With the onset of lunar mapping by the US Geological Survey, several years ago, lunar stratigraphy emerged as an interpretative tool of central importance in understanding the history of the Moon's surface. It is by no means the only effective means of access to an understanding of lunar geology, but it is one of the essential paths that can be investigated in a reasonably short time only by a team of lunar workers larger than is commonly encountered in, say, the universities. Dr E. Shoemaker led this mapping programme and directed much attention to a study of Mare Imbrium and the characteristic facies (some would say "deposits") that are associated with it and are found over a large part of the Moon's surface. Mutch expounds on the Imbrium stratigraphy and proceeds to describe the stratigraphies of other lunar maria and eraters, and then of volcanic flows and deposits on both Earth and Moon. He avoids one important problem concerned with the flow in Mare Imbrium (Fig. VIII-21), in which the number density of craters is appreciably higher on the most recent flow than on stratigraphically older units (one might expect the converse) by stating, in the caption, "Crater densities on both sides of the flow are similar. . .".

Interpretation of reported facts is minimal and is coloured by prevailing US Geological Survey views. Most of the viewpoints expressed will find general support; some of these ideas may change, but Mutch does not stick his neck out, and one of the values of his book is that it will remain a useful reference work. The chapter on highland stratigraphy, and the crisp impressions of lunar rocks in the photographs taken during and following the Apollo XI mission, highlight the terminal parts of the book: it should be read by students of the Moon.

\section{G. FIELDER}

\section{MAN AND THE SOLAR SYSTEM}

\section{The Atlas of the Universe}

By Patrick Moore. Pp. 272. (Mitchell Beazley with George Philip: London, September 1970.) $215 s$.

THrs is definitely a book for the coffee table rather than for the professional library, but the many excellent colour pictures of the Earth from various NASA surveys are alone worth the cost of the volume, and may perhaps ensure that it will be found on the coffee tables of professional institutions as well as those of the private individual. The rest of the book leaves much to be desired in view of its all-embracing title. It is not an atlas of the universe (let alone the definitive work suggested by the use of the definite article), any more than a book of the same length could claim to be a history of the world; it is only on page 188 that discussion of the stars is introduced (along with more excellent illustrations), and less than 10 per cent of the volume is devoted to discussing external galaxies.

It is unfortunate that the author has overreached himself in this way, because his product is much more impressive when considered as an atlas of the Solar System. The text adds nothing to the information available in other books with less extravagant titles, but the illustrations (both photographic and diagrammatic) are so profuse and of such a high standard that they are informative in themselves, and may encourage some readers to delve further into a study of astronomy and space science. Because of this, the atlas seems particularly suitable for schools.

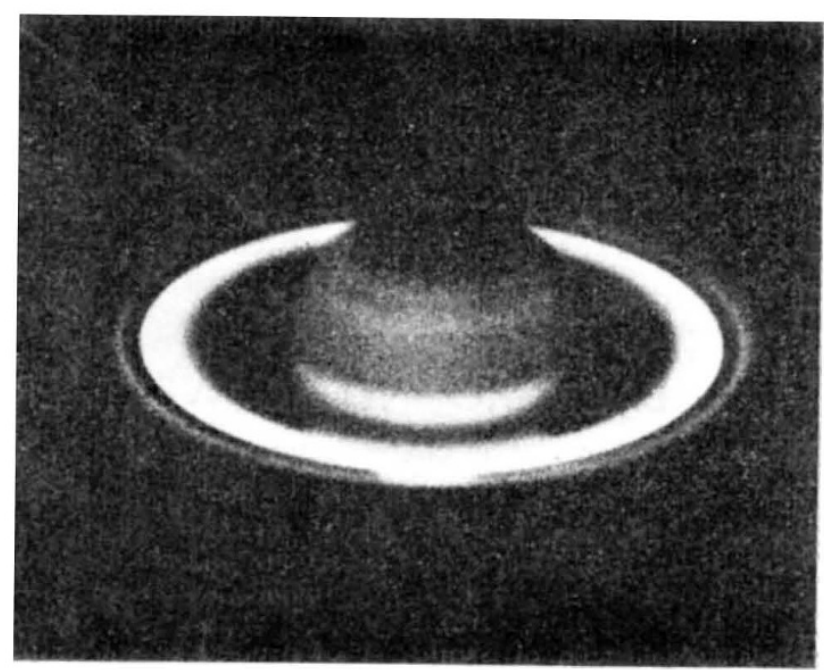

Saturn's rings, photographed by the Lowell Observatory, Flagstaff, Arizona (from The Atlas of the Universe).

Although I cannot agree with the packaging of the book, its contents are of undoubted worth, and would be even more commendable presented in the context of man's exploration of the Solar System, from which its. inspiration and much of its content are clearly drawn. The apparently belated recognition of this by the producers of the book makes the discrepancy between package. and contents still more irritating -if the epilogue by NASA's Dr Thomas Paine had been used as a prologue, and the title of the book suitably altered, it would have been wholly admirable. JOHN GRIBBIN

\section{IONOSPHERIC PHYSICS}

Propagation of ELF and VLF Waves near the Earth By Yakov L. Al'pert and Dora S. Fligel'. Translated from the Russian by James S. Wood. Translation edited by James R. Wait. Pp. v+171. (Consultants Bureau: New York and London, 1970.) \$22.50.

Thrs book represents a concise summary of several of the theoretical problems encountered in the study of waves in the frequency range $0.001<f<30 \mathrm{kHz}$ propagating in a bounded medium. The authors, in their introduction, suggest that they wish to give an insight on this branch of radiophysics to the inexperienced reader. In this they have been unsuccessful because many of the problems discussed are presented assuming that the reader has a strong background. As a reference text for the expert, however, this book contains a wealth of information pertaining to certain boundary value problems encountered in the propagation of electromagnetic waves in the ionosphere. In particular the authors give an excellent account of the evaluation of the roots of the pole equation, which does much to enhance the readability of the mathematical development. To acquire an adequate background before attacking this book, the reader should first study Al'pert's original book Radio Wave Propagation and the Ionosphere of which this new book is a natural offshoot. This would give the reader a chance to familiarize himself with Al'pert's style and notation, as well as giving him a feeling for the transition in thinking from the presatellite days of Al'pert's first book to the present time when satellites are capable of making in situ measurements of the wave phenomena which Al'pert studies theoretically. The reader benefits from a large number of comments by the editor (J. R. Wait). As the editor 